\title{
La ecología disciplinar y la intrusión de la problemática ambiental: hacia la "percepción de fenómenos globales"
}

\author{
Federico DI PASQUO \\ Becario doctora \\ dipasquof@yahoo.com.ar \\ UBA \\ Guillermo FoLGUERA \\ Becario postdoctoral \\ guillefolguera@yahoo.com.ar \\ CONICET \\ Alberto ONNA \\ Profesor adjunto \\ alberto.onna@gmail.com \\ UBA
}

Recibido: 24 de Enero de 2011

Enviado a evaluar: 25 de Enero de 2011

Aceptado: 5 de Octubre de 2011

\section{RESUMEN}

A partir de la crisis ambiental a comienzos de 1960, una serie de discursos provenientes de diferentes sectores sociales acordaron en su alcance global. Paralelamente, se consolidaron dos áreas de la ecología disciplinar: la ecología del paisaje y la macroecología. La pregunta general que enmarca al presente trabajo es: ¿cuál es la relación entre la problemática ambiental y la ecología disciplinar? Nuestra hipótesis principal es que la problemática ambiental efectivamente habría modulado el desarrollo de la ecología disciplinar. En particular, a partir de 1980 el discurso ambiental habría afectado a las investigaciones ecológicas incrementando sus escalas de estudio.

Palabras clave: crisis ambiental, ecología del paisaje, macroecología, escala espacial.

The ecology and the interference of the ambient problematic: toward the "perception of global phenomena"

\begin{abstract}
In the context of the beginning of 60's, series of discourses performed by different social groups agree with the global condition of the ambient crisis. In the same decade, two areas of knowledge born within ecology: landscape ecology and macroecology. The main question of this work is: what is the relationship between the ambient problem and disciplinary ecology? Our hypothesis is that ambient problem may modulate the development of ecology. In particular, one of the main conclusions is that since 80's ambient discourse may affect ecological investigations increasing its scales of studies.
\end{abstract}

Keywords: ambient crisis, landscape ecology, macroecology, spatial scale. 
Titre: écologie comme matière et intrusion de la problématique environnementale: vers "la perception des phénomènes globaux

\begin{abstract}
RESUMÉ
À partir de la crise environnementale au début des années 1960, toute une série de discours provenant de différents secteurs sociaux ont atteint un accord global. De manière parallèle, deux branches de l'écologie comme matière, sont consolidées: l'écologie du paysage et macroécologie. La question générale qui encadre le présent travail est la suivante: Quelle relation y a-t-il entre la problématique environnementale et l'écologie comme matière? Notre hypothèse principale est que la problématique environnementale a réellement influencé le développement de l'écologie comme matière. Notamment à partir de 1980, le discours environnemental a affecté aux recherches écologiques tout en augmentant ses échelles d'étude..
\end{abstract}

Mots clé: crise environnementale, écologie du paysage, échelle spatiale.

\title{
1. INTRODUCCIÓN
}

La relación entre la ecología disciplinar y la crisis ambiental fue analizada por numerosos autores de diferentes tendencias: Hagen 2008; Leff 2007; Kingsland 2004; Bowler 1998; Bocking 1995; Deléage 1993; Hagen 1992; Pimm 1991; Bramwell 1989; Morin 1989; Naess 1989; McIntosh 1982; Carson 1962; Leopold 1949. En el caso de estos últimos en general, el esfuerzo estuvo dirigido a entender qué lugar debió ocupar esta meta-disciplina ${ }^{1}$ frente a la crisis planteada:

Historians of science, in analyzing ecology as a scientific discipline, have addressed several aspects of its relation to environmental protection: the invocation by ecologists of practical relevance to justify increased funding; the common metaphors of ecology and technocratic approaches to managing human society; and the origins of ecological research tools in new environmental problem. (Bocking 1995, p. 1).

En este sentido, el abordaje de dicha relación fue "asimétrica", en la medida en que se evaluó mayormente el modo en que la ecología disciplinar podría afectar el desarrollo de la crisis ambiental. De este manera, no se registró por entonces una reflexión dirigida hacia el interior de esta meta-disciplina, más allá del propio reconocimiento de ciertos cánones comunes a todas las ciencias naturales (ej. mecanicismo, materialismo, progreso, racionalidad, objetividad,

${ }^{1}$ Con 'ecología disciplinar' nos referimos específicamente a la ecología científica, ámbito de la biología. Asimismo, también la denominaremos meta-disciplina debido a la multiplicidad de áreas del saber que la constituyen (ej. ecología del comportamiento, ecología de poblaciones, ecología de comunidades, ecología de ecosistemas, ecología del paisaje, macroecología, etc.). 
neutralidad) (Bowler 1998). Así, la pregunta general que enmarca al presente trabajo es: ¿la ecología disciplinar se vio modulada por la crisis ambiental? Y si esto es afirmativo, ¿de qué modo lo hizo?

Durante la crisis ambiental (comprendida desde los inicios de la década 1960 hasta la actualidad) se originaron y consolidaron dos áreas del saber, en el seno de la ecología disciplinar: la ecología del paisaje (Odum y Barrett 2008; Burel y Baudry 2004; Naveh y Liberman 2001; Farina 1998; Forman 1995a) y la macroecología (Witman y Kaustuv 2009; Brown 2003; Gaston y Blackburn 2000; Brown y Maurer 1989). Si bien ambas disciplinas difieren entre sí en diversos aspectos, tienen en común que sus investigaciones abarcan grandes áreas geográficas, esto es, indagan fenómenos que son relevantes en escalas espaciales (y temporales) ${ }^{2}$ amplias. En relación a ello, en este trabajo sostenemos las siguientes hipótesis:

I. Los discursos de la ecología del paisaje y de la macroecología se relacionan estrechamente con el discurso ambiental.

II. En particular, la relación se expresaría en lo que entenderemos como una "percepción de fenómenos globales" dentro de los discursos analizados. Esto es, atender a fenómenos que son relevantes en escalas espaciales amplias, o bien, áreas geográficas ${ }^{3}$ amplias. Ahora bien, dichos fenómenos pueden tratarse tanto de procesos como de patrones ecológicos (señalados en el discurso de las disciplinas ecológicas), o bien actividades del hombre que degradan el ambiente (señalados en el discurso ambiental) ${ }^{4}$.

A los fines de indagar esta problemática, el trabajo se organizó en tres secciones claramente delimitadas. En la próxima sección se realiza una breve reseña histórica del surgimiento y consolidación de las dos disciplinas analizadas: ecología del paisaje (apartado 2.1) y de la macroecología (apartado 2.2). En la siguiente sección se presentan tres temáticas. Por un lado, son revisadas algunas características de la ecología disciplinar hasta la década de 1980 (apartado 3.1), por otro lado, nos referimos al contexto "extra-disciplinar" representado por el discurso ambiental (3.2) y por último, señalamos un "obstáculo" entre las investigaciones ecológicas y las problemáticas ambientales (3.3). En el cuarto apartado se introduce la "percepción de fenómenos globales" dentro de la ecología disciplinar, en el contexto de la jerarquía espacial. Finalmente, se presentan algunas conclusiones generales.

2 Según Farina (1998), escala refiere a las dimensiones físicas espaciales (y temporales) de fenómenos observados

${ }^{3}$ La escala espacial es la dimensión física del área geográfica. En este sentido, un área geográfica "amplia" se corresponde con una escala espacial "amplia".

${ }^{4}$ No obstante, se debe considerar que muchos problemas ambientales se relacionan directamente con procesos ecológicos (ej. la fragmentación de hábitats por actividad humana y una consecuente disminución en la diversidad de especies). 


\section{EL SURGIMIENTO Y LA CONSOLIDACIÓN DE LA ECOLOGÍA DEL PAISAJE Y DE LA MACROECOLOGÍA}

\subsection{ORIGEN Y CONSOLIDACIÓN DE LA ECOLOGÍA DEL PAISAJE}

Desde 1960 hasta 1980 se gestó una de las áreas del saber de la ecología disciplinar, la ecología del paisaje. Las actividades en torno a dicha disciplina se iniciaron principalmente mediante investigaciones realizadas en Europa y, posteriormente, en Estados Unidos (Turner et al. 2001). Cabe mencionar que se presentaron interesantes diferencias tanto entre los conceptos básicos como en su aplicación práctica entre las investigaciones desarrolladas en estos dos lugares. Mientras que en Europa estos estudios buscaron dar respuesta a problemas concretos de planificación, conservación y uso de la tierra, en Estados Unidos los trabajos se enfocaron al desarrollo y dinámica de la heterogeneidad espacial de variables bióticas y abióticas y, en sus efectos sobre el patrón del paisaje (Matteucci 1998c). Hacia fines de 1960 dicha disciplina se afianzó cuando tuvo lugar en Alemania el primer simposio de la ecología del paisaje. Para la década de 1980, se originaron nuevos conceptos y avances dirigidos a la fragmentación de hábitat, la conservación de la diversidad, los corredores biológicos, la conectividad, y el desarrollo de métodos cuantitativos y estadísticos. Además, en esa década, se fundó la International Association of Landscape Ecology (IALE) junto a su publicación periódica Landscape Ecology, consolidando definitivamente dicha disciplina (Subirós et al. 2006; Naveh y Liberman 2001).

\subsection{ORIGEN Y CONSOLIDACIÓN DE LA MACROECOLOGÍA}

Avanzada la década de 1980, primero en investigaciones realizadas en Estados Unidos y posteriormente también en Inglaterra, se dio origen a otra disciplina de la ecología: la macroecología. La misma tuvo lugar a partir del interés en temáticas relacionadas, entre otras, con la biogeografía (Ruggiero 2001) y con la estructuración de las comunidades ${ }^{5}$ (Brown 2003). Trabajos pioneros como el de Hutchinson y Mac Arthur (1959), Mac Arthur (1972), Rapoport (1975), sentaron las bases donde se pueden enmarcar toda una línea de ensayos que continuó durante la década de 1980 (Brown 1981; Damuth 1981; Brown 1984; Damuth 1987; Brown y Maurer 1987; Morse et al. 1988). En 1989, se sistematizó formalmente la macroecología a través de un artículo de Brown y Maurer: Macroecology: the division of food and space among species on continents. Aquí los autores explicitaron el marco conceptual de dicha área (di Pasquo y Folguera 2009; Brown 2003; Gaston y Blackburn 2000). No obstante, esta nueva disciplina de la ecología se consolidó recién en 1991, cuando

\footnotetext{
${ }^{5}$ La estructura comunitaria se puede reflejar, por ejemplo: con el tamaño corporal, el desplazamiento de caracteres, distribuciones anidadas, etc. (Brown 2003).
} 
comenzó a publicarse la primera revista sobre la temática, Global Ecology and Biogeography. A Journal of Macroecology.

Es importante señalar, en relación a nuestros objetivos, el impacto que la ecología del paisaje y la macroecología tuvieron entre los ecólogos. En este sentido, Turner (2005) en el artículo Landscape Ecology in North America: Past, Present, and Future revisó el número de publicaciones anuales entre 1982 y 2003 que incluyen los términos 'ecología' y 'paisaje' o 'ecología del paisaje' en el titulo, en el resumen o en las palabras claves. Turner encontró un aumento exponencial en el uso de los términos a partir de 1990. A su vez, en relación a la macroecología, Smith y colaboradores (2008) en el artículo: Macroecology: more than the division of food and space among species on continents, revisaron el número de publicaciones entre 1988 y 2007 que incluyen el término 'macroecología' en el titulo, en el resumen o en las palabras claves. Los mismos, también encontraron un incremento exponencial del uso del término, alrededor de 1995. No se puede obviar que estas dos disciplinas de reciente aparición, penetraron fuertemente dentro de la ecología disciplinar en un periodo de tiempo notablemente breve. Ahora bien ¿a que se debió este "rápido" desarrollo? Entendemos que parte de la respuesta se encontró en la irrupción que tuvo la problemática ambiental sobre la ecología disciplinar.

A continuación, evaluaremos el contexto disciplinar (apartado 3.1) del cual emergen éstas áreas del saber, así como también el contexto generado a raíz de la crisis ambiental (3.2).

\section{ALGUNOS ASPECTOS DE LA ECOLOGÍA DISCIPLINAR Y DE LA CRISIS AMBIENTAL}

\subsection{EL USO DE ESCALAS “ANTROPOCÉNTRICAS” EN LA INTERPRETACIÓN DE LOS FENÓMENOS ECOLÓGICOS}

Desde la década de 1950 se forjó el marco conceptual que prevaleció en la ecología disciplinar hasta fines de la década de 1970. A ello, se le sumó una fuerte tendencia al abandono de estudios descriptivos siendo reemplazados por estudios experimentales (Nuñez et al. 2008). Resulta interesante destacar dos aspectos generales de las distintas investigaciones ecológicas realizadas durante ese periodo. El primero es que, hubo cierta tendencia a trabajar sobre ecosistemas definidos sobre áreas pequeñas, homogéneas y "naturales" (es decir, no antropizados) hasta la décadas de 1970 (Bailey 2009; Matteucci 1998c):

Until 1970, most major research programmes in ecology worked within the framework of the ecosystem defined as a homogeneous biocoenosis developing in a homogeneous environment. The main subjects of research were 'natural' systems such as the forest or the savannah. (Burel y Baudry 2004, p. 13). 
El segundo aspecto (más importante a los fines de nuestro trabajo) es que, hasta la década de 1980, las investigaciones ecológicas utilizaron una escala 6 "antropocéntrica" ("familiar" a la experiencia del ecólogo) (Cueto 2006; Reboratti 2000): "Ecologists deal with phenomena that are intuitively familiar, and we are therefore more likely to perceive and study such phenomena on anthropocentric scales that accord with our own experiences." (Wiens 1989, p. 385). En este sentido, Kareiva y Andersen (1988), mostraron que de cien experimentos en comunidades naturales, la mitad de ellos fueron realizados en parcelas de menos de 1 metro de diámetro ${ }^{7}$ (Cueto 2006; Pimm 1991; Wiens 1989). En relación a lo antes dicho, es interesante destacar que los principales programas de investigación de esta meta-disciplina (desarrollados entre 1950 y 1980) en general, analizaron procesos más relevantes a escalas finas que en amplias escalas espaciales ${ }^{8}$. Algunos de los programas de investigación más salientes de la ecología (en ese periodo), se desarrollaron a partir de propuestas como: la sistematización energética del ecosistema dada por Lindeman, en 1942 y el desarrollo de la ecología de ecosistemas ${ }^{9}$ dada por los hermanos Odum a

${ }^{6}$ En las investigaciones ecológicas: "La escala [...] queda definida a través de la dimensión del estudio y de su unidad de medición. En términos espaciales, la extensión es la superficie total donde se realizan las observaciones o experiencias, y el grano es el tamaño de la unidad de observación, de medición o el área abarcada por el experimento (Casenave et al. 2007, p.196).

${ }^{7}$ En los experimentos a campo, se colectaban datos a escalas finas (una hectárea o menos) extrapolando los resultados sobre amplias escalas, sin ser considerados los supuestos y/o las fuentes de error involucradas (Cueto 2006).

${ }^{8}$ Dado que los procesos no son dependientes de la escala: "no puede decirse que un proceso esté restringido a una escala determinada, puesto que un proceso simplemente 'actúa' (...) la competencia entre las plantas de una comunidad es la misma ya sea observada en un jardín o en toda la extensión de un bioma de pastizal: el proceso competitivo es simplemente el efecto negativo de una planta individual sobre otra, tanto a la escala local como a la escala regional. Sin embargo, algunos procesos parecen prevalecer sobre otros a una determinada escala, produciéndose patrones dependientes de la escala que permiten inferir que dichos procesos son más importantes que otros a esa escala. Es importante resaltar este punto: no es que los procesos dejen de actuar, sino que alguno (o algunos) de ellos se hace más importante a una cierta escala, disminuyendo entonces la importancia relativa de los otros" (Casenave et al. 2007, p. 203).

9 El ecosistema, se define como la comunidad biótica más su medio abiótico. Por lo tanto, dicha noción, no se compromete con una dimensión espacial. Así, un gran lago o un pequeño estanque pueden ser un ecosistema. En este sentido, la ecología de ecosistemas ha colaborado con la idea de una ecología a gran escala. Por ello "navío espacial Tierra" se convirtió en una metáfora para científicos y aquellos, no científicos preocupados por la salud del planeta (Hagen, 2008). Sin embargo, como mencionamos: "Historically, ecosystems have been defined as small, homogeneous areas or sites, such as a stand of trees or a meadow" (Bailey 2009, p. 10). Tal vez, ello se deba al énfasis de un enfoque funcional, "preocupado" por la interacción organismos-ambiente (la cual, puede ser registrada en pocos metros cuadrados y luego extrapolada): "El concepto del ecosistema es y debe ser vasto, siendo su principal función en el pensamiento ecológico la de subrayar las relaciones forzosas, la interdependencia y las relaciones causales, esto es, el acoplamiento de componentes para formar unidades funcionales” (Odum 1972, p. 7). 
partir de 1950 (Deléage 1993; Hagen 1992; Odum 1972); la formalización de Hutchinson del concepto de nicho ecológico ${ }^{10}$ (McIntosh 1982; Hutchinson 1981); los debates de diversidad-estabilidad desarrollados desde 1950 hasta 1960 (Ives 2005) o la competencia interespecifica y la relevancia de la exclusión competitiva en la estructuración de las comunidades naturales, dada en 1960 (y puesta en duda entre 1970 y 1980) (Nuñez et al. 2008; Kingsland 1991). Así, a través de las décadas, los ecólogos lograron cierto acuerdo respecto de cuáles eran los ejes centrales de esta meta-disciplina, poniendo el énfasis sobre aquellos procesos que son más relevantes en escalas "antropocéntricas". A esta tendencia, se le presentaron algunas excepciones como el desarrollo de la teoría de biografiaría de islas ${ }^{11}$ dada entre 1963 y 1967 por MacArthur y Wilson (McIntosh 1982) o trabajos pioneros como el de MacArthur (1972), Whittaker (1972) ${ }^{12}$ o Rapoport (1975).

A partir de la década de 1980, comenzó una revisión crítica de aquellas investigaciones que involucraron el estudio de fenómenos ecológicos a través, de escalas de trabajo pequeñas (Schneider 2001; Turner et al. 2001; Levin 1992; Wiens 1989). En este sentido, Kemp y colaboradores (2001), mediante el artículo denominado Scaledependence and the problem of extrapolation revisaron los trabajos publicados entre 1978 y 1997 que habían mencionado el término 'escala' en el resumen o en las palabras claves. Los mismos, encontraron una tendencia creciente en los estudios ecológicos que enfatizaban el uso de este concepto; poniendo en evidencia el impacto que tuvo la noción de escala en el periodo analizado. Antes de 1980 eran más bien escasos los trabajos que explicitaban la escala de estudio. Avanzada la década de 1980, se afianzó la idea de que los organismos y los procesos ecológicos operaban en un rango de escalas espaciales y temporales (Schneider 2001). De esta manera, se evidenció que los patrones, los procesos y las propiedades ecológicas (ej. la diversidad) estaban fuertemente asociados a la escala de observación ${ }^{13}$ (o escala de estudio) (Kemp et al. 2001). A la vez, se reconoció que los fenómenos ecológicos no deben ser indagados únicamente desde escalas "antropocéntricas".

${ }^{10}$ El concepto de nicho ecológico se deriva del principio de exclusión competitiva de Gause y de la ley de Malthus del crecimiento exponencial. El mismo, tiene como corolario que las especies que coexisten en un mismo ambiente tienden a diferir en sus requerimientos (Brown 2003).

11 Resulta interesante notar que la teoría de biogeografía de islas influyó en el desarrollo de la ecología del paisaje, es decir, se reconoce que una isla puede ser tratada como un parche de hábitat aislado y el océano como una matriz que presenta resistencia a los organismos (Odum y Barrett 2008; Subirós et al. 2006).

12 En éste ensayo Whittaker "introduce la jerarquía de diversidades, en la cual distintos tipos de diversidad (por ejemplo, alfa, beta o gama) están asociadas por definición a diferentes escalas espaciales, si bien no de manera explícita" (Casenave et al. 2007, p. 195).

13 Es importante destacar que los patrones pueden ser dependiente de la escala, mientras que los procesos no lo son. No obstante, "los patrones (...) que pueden ser detectados están determinados por la escala espacial y temporal de la investigación (y, en consecuencia, también lo estarán los procesos si estos son inferidos a partir de ellos)" (Casenave et al. 2007, p. 198). 
Es importante resaltar a los fines de las hipótesis presentadas que la disciplinas aquí analizadas (la ecología del paisaje y la macroecología), se diferenciaron por realizar sus investigaciones sobre grandes áreas geográficas o bien, amplias escalas espaciales. Apartándose fuertemente del tradicional enfoque que suponían las investigaciones en ecología (escalas finas y ecosistemas definidos sobre pequeñas áreas homogéneas).

A continuación, revisamos algunas de las coyunturas del contexto "extra-disciplinar" en el que tuvieron lugar las nuevas áreas aquí indagadas.

\subsection{LA CRISIS AMBIENTAL Y SU ALCANCE GLOBAL}

En este apartado analizaremos el discurso ambiental ya debatido extensamente en la bibliografía. Sin embargo, se pretende simplemente poner en evidencia una característica común a los debates ambientales: el discurso ambiental situó los problemas a tratar sobre un nivel global o bien regional. Entendemos que este "aspecto" es esencial para comprender el desarrollo de la ecología del paisaje y el de la macroecología, así como también la penetración del discurso ambiental en la ecología disciplinar.

Muchos debates transcurridos en el periodo de 1960-1990, se caracterizaron por situar los fenómenos a tratar sobre una escala global o regional. Por ejemplo: los modelos desarrollados a partir del Club de Roma (1968) donde se trataron los cambios que se estaban produciendo en el planeta a consecuencia de las acciones humanas (Onna et al. 2008; Gallopín 2004; Oteiza 2004; Meadows et al. 1972); o en los debates desarrolladas por los ecologistas a comienzos de 1970, donde se desplegó una lucha enfática defendiendo la "salud"del planeta y se evidenció la necesidad de un enfoque a gran escala que "excedió" las fronteras entre los países ${ }^{14}$ (ver principio 18) (Onna et al. 2009) y la crisis de los combustibles que alertó acerca de la finitud de los recursos terrestres durante 1973 y fines de 1974 (Yustos 2009; Scorer 1980; Grenon 1974). Otro ejemplo, fue la creación en 1988 del Panel Intergubernamental de Expertos sobre el Cambio Climático (IPCC). Según el IPCC estamos ante las primeras alteraciones climáticas planetarias de origen humano, motivadas por los gases de efecto invernadero (Yustos 2009). En otras palabras, las actividades humanas tienen lugar a una escala en la cual pueden interferir con sistemas naturales como el clima del planeta (Parry et al. 2007).

Fue en 1984, cuando se constituyó la Comisión Mundial sobre Medio Ambiente y Desarrollo con el objetivo de evaluar la degradación ambiental y la aplicación de las políticas ambientales. De aquí surgió el informe Bruntland, conocido también con el nombre, "Nuestro Futuro Común" (Leff 2007). Sobre la base de dicho informe y como respuesta a la penetración del discurso ecologista, la crisis energética y la capacidad de carga del planeta, entre otros; se convocó, a la Conferencia de las Naciones Unidas sobre Medio Ambiente y Desarrollo, en Río de Janeiro (1992). En la misma,

\footnotetext{
14 Es importante destacar que a través, de la propaganda y la protesta esté movimiento repercutió dentro de la sociedad, generando un "discurso ecologista" que penetro en las agendas de las clases políticas y empresariales dando lugar a un cambio de las prioridades (Prado 1996).
} 
asistieron más de cien jefes de Estado e importantes empresarios, donde declararon su compromiso con la "salud" del planeta. Entre los principios de la declaración de Río podemos señalar el $\mathrm{n}^{\circ} 7$ :

Los Estados deberán cooperar con espíritu de solidaridad mundial para conservar, proteger y restablecer la salud y la integridad del ecosistema de la Tierra. En vista de que han contribuido en distinta medida a la degradación del medio ambiente mundial [...]. (Grinberg 1999, p. 198).

Así como el principio n ${ }^{\circ} 18$ : "Los Estados deberán notificar inmediatamente a otros Estados de los desastres naturales u otras situaciones de emergencia que puedan producir efectos nocivos súbitos en el medio ambiente de esos Estados [...]." (Grinberg 1999, p. 198). A partir de la declaración de Río, quedó establecido que la "salud" del planeta debe ser atendida por los diferentes Estados reconociéndose que la crisis ambiental atañe a todo el globo. Así, para la década de 1990 se alcanzó cierto consenso respecto de la situación ambiental, es decir, se reconoció el deterioro del planeta y la "globalización" de los problemas ambientales (Matteucci 1998a). De esta manera, la situación ecológica crítica con repercusiones a gran escala se convirtió en una realidad tangible, es decir, se volvió uno de los aspectos más relevantes de la historia del siglo XX (Yustos 2009; Reboratti 2000; Puebla 1999). Resulta interesante destacar, a los fines de nuestras hipótesis, que la fórmula global de la problemática ambiental fue exitosa (Cornejo et al. 2001). De aquí, que se pueda reconocer dentro del discurso ambiental lo que nosotros denominamos "percepción de fenómenos globales".

\subsection{LA DISPARIDAD DE LA ESCALA: UN “OBSTÁCULO” ENTRE LAS INVES- TIGACIONES ECOLOGICAS Y LAS PROBLEMATICAS AMBIENTALES}

En este punto, resulta interesante detenerse a evaluar lo analizado hasta aquí. Según Bramwell (1989), el termino 'ecología' usado en un sentido normativo (y no en un sentido biológico), se relacionó con la conservación de los sistemas naturales. Donde cualquier modificación que "sufra" un sistema natural es visto como algo esencialmente negativo. La autora señala que esas modificaciones pueden ser relevadas sobre amplias escalas o bien, a un nivel que pueda afectar a la continuidad de la existencia humana. Mientras que la 'ecología' como ámbito de la biología, es aquella que estudia procesos dentro de ecosistemas cerrados definidos sobre pequeñas áreas. Coincidiendo con Bramwell, mostramos que los discursos revisados en los apartados (3.1) y (3.2) refieren en efecto a escalas distintas. Es decir, mientras que la ecología disciplinar se caracterizó por indagar fenómenos (patrones y procesos ecológicos) que son más relevantes a escalas pequeñas (al menos hasta la década de 1980); el discurso ambiental, indagó fenómenos (actividades del hombre que degradan el ambiente) que tienen lugar sobre amplias regiones geográficas. Esta disparidad fue señalada oportunamente por Pimm (1991):

The most pressing ecological problems involved many species and their fate across decade to centuries, over large geographical areas [...] Most ecological research is done on short time scales and over small spatial scale, and even community ecology typically involves few species [...] (pág. 1). 
Ahora bien, sí resulta cierto que la investigación ambiental debe tener bases científicas sólidas y, esas bases, en gran medida se espera que sean generadas por parte de la ecología disciplinar (Kingsland 2004). Entonces, se pone en evidencia el siguiente "obstáculo":

Aunque se pretenda aparentar que las decisiones sobre el futuro de nuestro planeta se fundamentan en los trabajos de la comunidad científica, tal percepción no corresponde con la realidad: [...] Más del 25\% de los estudios publicados en el Journal of Ecology de 1980 a 1986 se realizaron a escalas menores a $0.25 \mathrm{~m}$ de diámetro [...]. Sin embargo, estas investigaciones son utilizadas para realizar inferencias sobre fenómenos ecológicos que se suceden en áreas correspondientes a miles de $\mathrm{Km}^{2}[\ldots]$ ¿Cómo es posible, entonces, representar procesos globales partiendo de estudios con escalas tan pequeñas? (Lozano et al. 2007, pp. 33-34).

En la próxima sección, indagaremos cómo se disuelve el "obstáculo" arriba señalado, a partir de la consolidación de la ecología del paisaje y de la macroecología. A su vez, se indagará la penetración que el discurso ambiental tuvo dentro de la ecología disciplinar.

\section{LA ECOLOGÍA DEL PAISAJE Y LA MACROECOLOGÍA: HACIA LA "PERCEPCIÓN DE FENÓMENOS GLOBALES" DENTRO DE LA ECOLO- GÍA DISCIPLINAR}

Cuando observamos algún fenómeno de interés, nos ubicamos dentro de un continuo de dimensiones físicas, que va desde las dimensiones más pequeñas (escalas chicas o escalas finas) hasta las más grandes (escalas amplias o gran escala) (Matteucci 1998c). De este modo, el lugar en donde nos ubiquemos dentro de ese continuo es arbitrario, es decir, el observador elige (en la medida de lo posible) desde qué escala aproximarse a determinado fenómeno. Algo similar ocurre en las disciplinas, las cuales "eligen" desde que escala abordar los fenómenos que estudian y buscan allí sus explicaciones (Reboratti 2000; Matteucci 1998b). En este sentido, cada disciplina se acerca a los fenómenos que le interesan desde una escala específica (o un conjunto de escalas determinadas). La biología molecular, por ejemplo, requiere de instrumentos que "amplifiquen" sus objetos de estudio dado que trabaja sobre dimensiones físicas muy pequeñas. Mientras que otras áreas del saber, necesitan "achicar" los objetos de estudio para poder abordar el "conjunto", tal como el caso de la geografía y el uso de mapas (Matteucci 1998c). En este sentido, la ecología disciplinar parece haber "optado" por una escala de trabajo para sus investigaciones ecológicas; no obstante, la ecología del paisaje y la macroecología son disciplinas que adoptaron escalas de trabajo hasta ahora inéditas dentro de esta meta-disciplina. En relación a lo anteriormente mencionado, resulta interesante preguntarse ¿qué tienen en común la ecología del paisaje y la macroecología que las diferencia del resto de las disciplinas que también forman parte de la ecología científica? Para dar respuesta a nuestra pregunta, examinamos la jerarquía espacial (Bailey 2009; Forman 1995a). La misma, se vincula con la idea de que el terreno 
puede ser delimitado en áreas similares ${ }^{15}$ a diferentes escalas espaciales. En este sentido, dicha jerarquía postula patrones sobre el terreno relativamente estables para un conjunto de escalas espaciales (Forman 1995a). Por ejemplo: el ecosistema (un área homogénea de pocas hectáreas), el paisaje (entendido como una combinación heterogénea de ecosistemas locales y con una extensión de 10 a $100 \mathrm{Km}^{2}$ aproximadamente), la región (entendida como una combinación de paisajes), el continente o el planeta (Bailey 2009; Matteucci 1998b). Aquí, es importante distinguir entre la dimensión física (la escala) y el terreno (la porción del suelo). Así, la dimensión física de un ecosistema es en general la escala local, de igual modo la dimensión física de un paisaje es la escala de paisaje o en otro ejemplo, la dimensión física de todo el planeta es la escala global (Figura 1).

Figura 1. Jerarquía espacial. En la misma se puede observar el anidamiento: [escala local] escala de paisaje] escala regional] escala continental] escala global]. Donde un área pequeña por ejemplo, el ecosistema (a escala local) se encuentra totalmente encerrado por un área mayor, el paisaje (a escala de paisaje) (Bailey 2009).

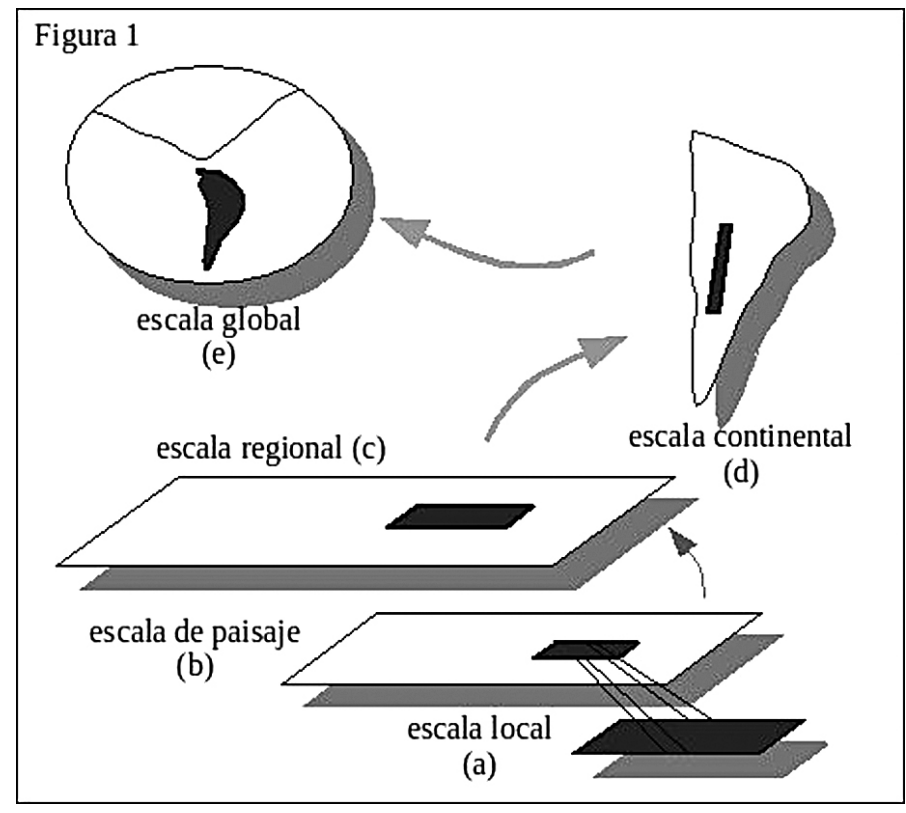

Ahora bien, si tomamos como referencia la jerarquía espacial, se pone en evidencia que tanto la ecología del paisaje como la macroecología descansan mayormente sobre las escalas superiores de la jerarquía (Naveh y Liberman 2001; Mateucci

15 El criterio para diferenciar éstas áreas, involucra: la vegetación, el perfil de suelo, la topografía, la geografía, la precipitación, regímenes de temperatura, etc. (Bailey 2009). 
1998b). En términos históricos, el análisis de los niveles superiores de la jerarquía espacial es algo novedoso dentro de la ecología disciplinar, dado que hasta la década de 1970 el ámbito de estudio "tradicional" de esta meta-disciplina fue el ecosistema, generalmente definido como pequeñas áreas homogéneas (figura 1: (a)) (Bailey 2009). Asimismo, hasta la década de 1980, las investigaciones ecológicas utilizaron una escala "antropocéntrica". Es decir, todas las disciplinas que integran ésta área del conocimiento trabajaron (y trabajan) sobre la escala local (o bien, sobre escalas más pequeñas). Sin embargo, con la consolidación de la ecología del paisaje en la década de 1980 y de la macroecología en los primeros años de la década de 1990, se comienzan a privilegiar otras escalas de trabajo. Por un lado, la escala de paisaje y la escala regional en la ecología del paisaje (Figura 1: (b) y (c)). Con relación a ello, Forman (1995b) menciona: "Just as full understanding of a liver or a town requires information from both broader and finer scales, understanding a landscape requires information on the broader region and on the finer-scale local ecosystems." (p. 134). Por otro lado, la macroecología - encargada de analizar patrones estadísticos emergentes de biotas que ocupan grandes áreas geográficas- privilegió la escala regional, continental e incluso la escala global (Brown y Maurer 1989) (figura 1: (c), (d) y (e)):

La macroecología tiende a enfocarse en fenómenos a escalas espaciales regionales o globales [...] Pero esto quiere decir que la macroecología frecuentemente se refiere a patrones y procesos en escalas mucho mayores que los pequeños sitios de estudio [es decir, la escala local] y cortas temporadas de campo de la mayoría de los ecólogos experimentales (Brown 2003, p. 39).

La incorporación de estas escalas espaciales dentro de los análisis ecológicos supone entonces que ciertos fenómenos (patrones y procesos ecológicos) deben ser abordados desde dimensiones físicas que están por encima de la escala local. En resumen, ambas disciplinas estudian fenómenos que son más relevantes sobre grandes áreas geográficas; evidenciándose esto en la jerarquía espacial ${ }^{16}$ y dando lugar a lo que nosotros reconocemos como "percepción de fenómenos globales". Resulta interesante resaltar a los fines de nuestro trabajo, que muchos ecólogos de ambas disciplinas y de otras áreas de la ecología coinciden, en que la interpretación de fenómenos ecológicos registrados sobre amplias escalas espaciales parecen recibir mayor atención, debido al reconocimiento de la problemática ambiental de carácter global (Odum y Barrett 2008; Smith et al. 2008; Turner 2005; Neilson 2005; Burel y Baudry 2004; Naveh y Liberman 2001; Turner et al. 2001; Matteucci 1998c; Farina 1993; Levin 1992; Turner 1990).

16 La jerarquía espacial es un desarrollo teórico más vinculado a la ecología del paisaje que a la macroecología. No obstante, la misma sirve de referencia para notar como dichas disciplinas indagan fenómenos que son relevantes en escalas espaciales amplias. 


\section{CONCLUSIÓN}

A través de este recorrido, hemos señalado que la ecología disciplinar (apartado 3.1) y el discurso desarrollado a partir de la crisis ambiental (3.2) se han referido a escalas distintas. Así, desde la década de 1950 hasta la década de 1980, la ecología científica ha desarrollado sus investigaciones sobre escalas finas y ecosistemas homogéneos definidos en áreas pequeñas; mientras que el discurso ambiental se ha referido a escala globales o bien, a grandes extensiones geográficas (3.3). A nuestro entender, este "obstáculo" moduló el "rápido" desarrollo y consolidación de disciplinas ecológicas que descansan sobre escalas espaciales amplias ${ }^{17}$; la ecología del paisaje en la década de 1980 y unos años después, la macroecología. A partir de aquí, es posible trazar un paralelismo entre las escalas utilizadas por la ecología disciplinar (ej. escala local, escala de paisaje, escala regional, escala continental y escala global) y las escalas a las cuales se plantean las problemáticas ambientales: "existe una jerarquía de problemas ambientales que ejercen influencia a nivel planetario, regional y local" (Cornejo et al. Pág. 111, 2001). Sin embargo, lejos de poder "conectar" soluciones desde la ecología disciplinar hacia las problemáticas ambientales, destacamos que el discurso ambiental refiere a fenómenos vinculados con actividades del hombre que degradan el ambiente, mientras que la ecología disciplinar indaga otro tipo de fenómenos, es decir, patrones y procesos ecológicos. Con ello, se busca evidenciar que estrictamente los problemas ambientales no toman la "forma" de problemas científicos, sino que se introducen oblicuamente a la ecología disciplinar (Pardo 1996). No obstante, no se puede obviar que el "común denominador" de estos fenómenos es su dimensión física. De este modo, entendemos que desde la ecología disciplinar, se busca "disolver" el obstáculo señalado respondiendo (en parte) a la demanda ambiental global.

A su vez, señalamos que la ecología del paisaje y la macroecología se abren paso dentro de la ecología disciplinar en un periodo en donde las bases conceptuales de esta meta-disciplina se estaban revisando. El interés por la noción de escala, a partir de la década de 1980, junto al reconocimiento de que los procesos y los patrones ecológicos se encuentran fuertemente asociados a la escala de observación fueron determinantes para el desarrollo de ambas disciplinas; las cuales suponen un cambio de escala notable para sus investigaciones. No obstante, la incorporación de amplias escalas de trabajo a la ecología científica plantea una serie de inconvenientes. Por un lado, la teoría ecológica clásica centrada en el estudio de relaciones entre pares de especies (o un grupo reducido de éstas) (ej. competencia, predación, mutualismo, parasitismo, cooperación, redes tróficas, etc.) (Margalef 1991) se pensaron para explicar fenómenos ecológicos (procesos) que parecen ser más preponderantes a escalas locales. Es decir, difícilmente estos marcos teóricos sean relevantes sobre amplias escalas espa-

17 Ello no significa que las disciplinas mencionadas no puedan desarrollar investigaciones a escala local. En efecto, en la actualidad ambas disciplinas desarrollan investigaciones que involucran todo el "espectro" de la jerarquía espacial. 
ciales (o por lo menos, no serian los únicos relevantes). Ello se debe a que los procesos ecológicos operan dentro de un rango espacio-(temporal) (Burel y Baudry 2004; Turner et al. 2001; Urban et al. 1987; Forman 1995a; Delcourt et al. 1988). Por lo tanto, aquellos procesos que "actúan" con mayor preponderancia dentro de un rango de escalas, disminuyen la importancia relativa de otros procesos actuantes (dentro de ese rango) (Casenave et al. 2007). Así, a escala local pueden ser preponderantes los procesos que involucran interacciones entre especies, mientras que a escalas mayores pueden ser más relevante otros procesos biológicos o procesos físicos ${ }^{18}$ (Delcourt et al. 1988). En general, a escalas amplias los procesos físicos disipan a los procesos biológicos (Wiens 1989). En este sentido, la ecología del paisaje y la macroecología enfocan parte de sus esfuerzos en comprender procesos que, en general, tienen más relevancia a escalas espaciales amplias (ej. extinción, especiación, cambios en la distribución geográfica, urbanización, el desarrollo de la agricultura, la fragmentación del hábitat) (Marrubio 2004; Brown 2003). Por otro lado, en el plano metodológico, se presenta una imposibilidad práctica para realizar experimentos controlados sobre grandes extensiones geográficas, tanto en la ecología del paisaje (Wu y Hobbs 2002; Hargrove y Pickering 1992) como en la macroecología (Blackburn 2004; Brown 2003). Estas consecuencias, tienen fuertes repercusiones dentro de la ecología disciplinar que obligan a repensar tanto aspectos epistemológicos como metodológicos de la misma (Wu y Hobbs 2002). De este modo, la problemática ambiental se "cuela" desde las exterioridades de la biología a los "paradigmas" de la ecología científica, a través de la dimensión física de los fenómenos indagados; barriendo certezas, abriendo nuevas preguntas y cuestionando el método experimental legado del modelo de la física (Leff 2007 y 2006).

Finalmente, concluimos que tanto el discurso ambiental como el dado por las disciplinas ecológicas indagadas, básicamente "consideran" fenómenos preponderantes en amplias escalas espaciales. Es decir, en ambos discursos se reconoce, lo que nosotros hemos denominado "percepción de fenómenos globales". Aparentemente, la crisis ambiental con su "carácter global" dio lugar a un nuevo lenguaje y "código cultural" que "atravesaron" a la sociedad occidental (Prado 1996). La cuál, penetró dentro de la ecología científica modulando a ciertas áreas de ésta meta-disciplina también hacia una "percepción global". Es decir, la ecología del paisaje y la macroecología, trabajan sobre escalas de estudio que son "más a fin" a la problemática ambiental que a la propia ecología disciplinar.

${ }^{18}$ El grano y la extensión que caracterizan a la escala en las investigaciones ecológicas son “[...] aspectos metodológicos [que] se trasladan al ámbito conceptual cuando se hace referencia a [...] procesos asociados a una escala en función del ámbito espacial y temporal que es abarcado por ellos. El fenómeno El Niño, por ejemplo, [un procesos físico] está asociado a una escala espacial que comprende entre 105 Y 107 m (la mínima y máxima extensión lineal de costa afectada, respectivamente)" (Casenave et al. 2007, p.197). 


\section{BIBLIOGRAFÍA}

BAILEY, R. G. Ecosystem Geography. From Ecoregions to Sites. Springer New York. London. 2009.

BLACKBURN, T. M. Method in macroecology. Basic and Applied Ecology. 5 (5), pp. 401-412. 2004.

BOCKING, S. Ecosystems, Ecologists, and the Atom: Environmental Research at Oak Ridge National Laboratory. Journal of the History of Biology. 28 (1), pp. 147. 1995.

BOWLER, P. J. Historia Fontana de las Ciencias Ambientales. Fondo de Cultura Económica. México. 1998.

BRAMWELL, A. (1989). Ecology in the twentieth century: a history. Yale University Press. New Haven and London. 1992.

BROWN, J. H. Two decades of homage to Santa Rosalia: toward a general theory of diversity. American Zoology. 21 (4), pp. 877-888. 1981.

BROWN, J. H. On the relationship between abundance and distribution of species. The American Naturalist. 124 (2), pp. 255-279. 1984.

BROWN, J. H. Macroecología. Fondo de Cultura Económica. Ed. Medellín, R. México.2003.

BROWN J. H. \& MAURER, B. A. Evolution of species assemblages: effects of energetic constraints and species dynamics on the diversification of the North American avifauna. American Naturalist. 130 (1), pp. 1-17. 1987.

BROWN J. H. \& MAURER, B. A. Macroecology: the division of food and space among species on continents. Science. 243 (4895), pp. 1145-1150. 1989.

BUREL, F. \& BAUDRY, J. (1999). Landscape Ecology Concepts, Methods and Applications. Science Publishers. United States of America. 2004.

CARSON, R.(1962). La primavera silenciosa. Crítica. España, Barcelona. 2005.

CASENAVE, L. J.; MARONE, L.; CAMUS, P. A. \& JAKSIC, E. M. "Escalas" en Jaksic, E. M. \& Marone, L. (eds) (2001), Ecología de Comunidades. Ediciones Universidad Católica de Chile. Chile, Santiago. 2007.

CORNEJO, R., VIANA, M. L. \& QUINTANA, M. "Cambio global: perspectiva ético-epistemológica" en Caracciolo, R. \& Letzen, D. (eds) (2001) Epistemología e Historia de la Ciencia. Universidad Nacional de Córdoba. Argentina. Vol. 7. 2001.

CUETO, V. R. Escalas en ecología: su importancia para el estudio de la selección de hábitat en aves. Hornero. 21 (1), pp. 1-13. 2006.

DAMUTH, J. Population density and body size in mammals. Nature. (290), pp. 699700. 1981.

DAMUTH, J. Interspecific allometry of population density in mammals and other animals: The independence of body mass and population energy-use. Biological Journal of the Linnean Society. (31), pp. 193-246. 1987.

DELCOURT, H. R. \& DELCOURT, P. A. Quaternary landscape ecology: Relevant scales in space and time. Landscape Ecology. 2 (1), pp 23-44. 1988.

DELÉAGE, J. P. Historia de la ecología. ICARIA. Barcelona. 1993. 
DI PASQUO, F. M. \& FOLGUERA, G. Tres dimensiones del reduccionismo en el contexto de la Teoría Metabólica Ecológica. Principia, an international journal of epistemology. 13 (1), pp. 51-65. 2009.

FARINA, A. Principles and methods in Landscape Ecology. Cambridge University Press. London. 1998.

FORMAN, R. T. T. Land Mosaics. The ecology of landscape and regions. Cambridge University Press. United Kingdom. 1995a.

FORMAN, R. T. T. Some general principles of landscape and regional ecology. Landscape Ecology. 10 (3), pp. 133-142. 1995b.

GALLOPÍN, G. C. "El Modelo Mundial Latinoamericano ("Modelo Bariloche"): Tres décadas atrás" en Herrera, A. O. \& otros (eds) (2004) ¿Catástrofe o Nueva Sociedad? Modelo Mundial Latinoamericano. 30 años después. Centro Internacional de Investigaciones para el Desarrollo. Canadá,Ottawa. pp.13-26. 2004.

GASTON, K. J. \& BLACKBURN, T. M. Pattern and Process in Macroecology. Blackwell Science. United Kingdom. 2000.

GRENON, M. La crisis mundial de la energía. Alianza Editorial. España, Madrid. 1974.

GRINBERG, M. Ecofalacias. De cómo las multinacionales se apoderan del discurso del ambiente. Galerna. Argentina, Buenos Aires 1999.

HAGEN, J. B. An Entangled Bank: The Origins of ecosystem Ecology. Rutgers University Press. New Brunswick, New Jersey. 1992.

HAGEN, J. B. Teaching Ecology during the Environmental Age, 1965-1980. Environmental History. (13), pp. 704-723. 2008.

HARGROVE W. W. \& PICKERING, J. Pseudoreplication: a sine qua non for regional ecology. Landscape Ecology. 6 (4), pp. 251-258. 1992.

HUTCHINSON, G. E. \& MACARTHUR R. H. A theoretical ecological model of size distributions among species of animals. American Naturalist. 93 (869), pp. 117-125. 1959.

HUTCHINSON, G. E. (1978). Introducción a la ecología de poblaciones. Blume Editorial. España, Barcelona. 1981.

IVES, A. R. "Community diversity and stability: Changing perspetives definitions" en Cuddington, K. \& Beisner, B. (eds) (2005) Ecologycal paradigms lost. Routes of theory change. Elsevier Academic Press. United States of America. 2005.

KAREIVA, P. \& ANDERSEN, M. Spatial aspects of species interactions: The wedding of models and experiments. Lecture Notes in Biomathematics. (77), pp. 35-50. 1988.

KEMP, W. M., PETERSEN, J. E. \& GARDNER, R. H. "Scale-dependence and the problem of extrapolation. Implications for Experimental and Natural Coastal Ecosystems" en Gardner, R. H., Kemp, W. M., Petersen, J. E. \& Kennedy, V. S. (eds) (2001) Scaling relations in experimental ecology. Columbia University Press. New York. 2001.

KINGSLAND. E. S. "Defining Ecology as a Science" en Real, L; Brown, J (eds) (1991) Foundations of Ecology. Classic Papers with Commentaries. University of Chicago Press. Chicago. 1991.

KINGSLAND. E. S. Conveying the intellectual challenge of ecology: an historical perspective. Frontiers in Ecology and the Environment. 2 (7), pp. 367-374. 2004. 
LEFF, E. Aventuras de la epistemología ambiental. Siglo XXI. México. 2006.

LEFF, E. (1998). Saber ambiental. Sustentabilidad, racionalidad, complejidad, poder. Siglo XXI. México. 2007.

LEOPOLD, A. (1949). "La ética de la Tierra" en Valdés, M. (ed) Naturaleza y Valor. Una aproximación a la ética ambiental. Fondo de Cultura Económica. México. pp. 25-44. 2004.

LEVIN, S. A. The Problem of Pattern and Scale in Ecology. Ecology. 73 (6), pp. 1943-1967. 1992.

LINDEMAN, R. L. The trophic-dynamic aspect of ecology. Ecology. (23), pp. 399418. 1942.

LOZANO, J. A. A., MILÁN, P. M., ROBlEDO, M. A. \& CARAVEO, L. N. Amenaza previsible. Lecciones de historia sobre la aplicabilidad del principio precautorio. Trayectorias, $\mathrm{n}^{\circ} 24.2007$.

MACARTHUR, R. H. Geographical Ecology. Patterns in the Distribution of Species. Harper \& Row, Publishers. New York. 1972.

MACARTHUR, R. H \& WILSON, O. E. An equilibrium theory of insular zoogeography. Evolution. 17 (4), pp. 373-387. 1963.

MARGALEF, R. (1991). Teoría de los sistemas ecológicos. Universitat de Barcelona. Barcelona. 1993.

MARRUBIO, M. P. La percepción remota y la tecnología SIG: una aplicación en Ecología de Paisaje. GeoFocus. 4, pp. 1-24. 2004.

MATTEUCCI, S. D. "La creciente importancia de los estudios del medio ambiente" en Matteucci, S. D. \& Buzai, G. D. (eds) (1998) Sistemas ambientales complejos: herramientas de análisis espacial. Eudeba. Argentina, Buenos Aires 1998a.

MATTEUCCI, S. D. "El análisis regional desde la ecología" en Matteucci, S. D. \& Buzai, G. D. (eds) (1998) Sistemas ambientales complejos: herramientas de análisis espacial. Eudeba. Argentina, Buenos Aires 1998b.

MATTEUCCI, S. D. "La cuestión del patrón y la escala" en Matteucci S. D. \& Buzai, G. D. (eds) (1998) Sistemas ambientales complejos: herramientas de análisis espacial. Eudeba. Argentina, Buenos Aires. 1998c.

McINTOSH, R. P. "The background and some current problems of theoretical ecology" en Saarinen, E. (ed) (1982) Concetual issues in ecology. D. Reídle Publishing Company, Dordrecht. Holanda. pp.1-61. 1982.

MEADOWS, D. H., MEADOWS, D. L., RANDERS, J. \& BEHRENS III, W. W. Los límites del crecimiento. Informe al Club de Roma sobre el Predicamento de la Humanidad. Fondo de Cultura Económica. México. 1972.

MORIN, E. (1989). "El pensamiento ecologizado" en Morin, E. \& Hulot, N.(eds) El año I de la era ecológica. Paidós. España. pp. 25-50. 2008.

MORSE, D.R., STORK, N.E. \& LAWTON, J.H. Species number, species abundance and body length relationships of arboreal beetles in Bornean lowland rain forest trees. Ecol. Entomol. 13, pp. 25-37. 1988.

NAESS, A. (1989). "La crisis del medio ambiente y el movimiento ecológico profundo" en Valdés, M. (ed) Naturaleza y Valor. Una aproximación a la ética ambiental. Fondo de Cultura Económica. México. pp. 25-44. 2004. 
NAVEH, Z. \& LIBERMAN, A. S. Ecología de Paisajes. Teoría y Aplicación. Facultad de Agronomía (UBA). Argentina, Buenos Aires. 2001.

NEILSON, R. P. "Landscape ecology and global change" en Wiens, J. y Moss, M. (eds) (2005) Issues and Perspectives in Landscape Ecology. Cambridge University Press. United Kingdom. 2005.

NÚÑEZ, P. G., NÚÑEZ, C. I. \& NÚÑEZ, A. M. La ecología en tensión. Una indagación histórica del presente de la disciplina. Observatorio Medioambiental. 11, pp. 13-24. 2008.

ODUM, E. Ecología. Interamericana. México. 1972.

ODUM, E. \& BARRETT, G. W. (1953). Fundamentos de Ecología. Cengage Learning Editores, S. A. México. 2008.

ONNA A., MONSERRAT A. L. \& DI PASQUO F. M. "Influencia de la crisis ambiental en la institucionalización de la Ecología argentina en la década de los '70s del siglo XX" en Faas, H. \& Severgnini, H. (eds) (2008) Epistemología e Historia de la Ciencia. Universidad Nacional de Córdoba. Argentina.Vol. 14. 2008.

ONNA A, MONSERRAT A. L, DI PASQUO F. M \& FOLGUERA G. La implementación de las áreas naturales protegidas entre dos tradiciones enfrentadas. Episteme, uma revista brasileira de filosofia e história das ciências. Aceptado. 2009.

OTEIZA, E. "El Modelo Mundial Latinoamericano: scriptum-post scriptum" en Herrera, A. O. \& otros (eds) (2004) ¿Catástrofe o Nueva Sociedad? Modelo Mundial Latinoamericano. 30 años después. Centro Internacional de Investigaciones para el Desarrollo. Canadá, Ottawa. pp. 7-12. 2004.

PARRY, M., CANZIANI, O., PALUTIKOF, J., VAN DER LINDEN, P. \& HANSON, C. (eds). Climate Change. Impacts, Adaptation and Vulnerability. Cambridge University Press. Cambridge, New York, Melbourne, Madrid, Cape Town, Singapore, São Paolo, Delhi. 2007.

PIMM, S. L. The balance of Nature? Ecological issues in the conservation of species and communities. The Chicago University Press. Chicago. 1991.

PRADO, M. M. Apuntes sobre la reflexividad en el movimiento ecologista. Política y Sociedad. 23, pp. 153-172. 1996.

PUEBLA, J. G. Cambio y persistencia en el espacio geográfico: consideraciones para la reflexión medioambiental. Observatorio Medioambiental. 2, pp. 25-39. 1999.

REBORATTI, C. Ambiente y sociedad. Conceptos y relaciones. Ariel. Argentina, Buenos Aires. 2000.

RAPOPORT, E. H. Areografía. Estrategias Geográficas de las Especies. Fondo de Cultura Económica. México. 1975.

RUGGIERO, A. "Interacciones entre la biogegrafía ecológica y la macroecología: Aportes para comprender los patrones espaciales en la diversidad biológica" en Bousquets, J. L. 1. \& Morrone, J. J. (eds) Introducción a la biogeografia en Latinoamérica: Teorías, conceptos, métodos y aplicaciones.UNAM. México. 2001.

SCORER, S. S. El idiota espabilado. Lo verdadero y lo falso en la catástrofe ecológica. Blume Editorial. España, Barcelona. 1980.

SCHNEIDER, D. C. The Rise of the Concept of Scale in Ecology. BioScience. 51 (7), pp. 545-554. 2001. 
SMITH, F. A., LYONS, S. K., ERNESt, S. K. M. \& BROWN, J. H. Macroecology: more than the division of food and space among species on continents. Progress in Physical Geography. 32 (2), pp. 115-138. 2008.

SUBIRÓS, J. V., LINDE, D. V., PASCUAL, A. L. \& PALOM, A. R. Conceptos y métodos fundamentales en ecología del paisaje (landscape ecology). Una interpretación desde la geografía. Doc. Anàl. Geogr. 48, 151-166. 2006.

TURNER, M. G. Spatial and temporal analysis of landscape patterns. Landscape Ecology. 4 (1), pp. 21-30. 1990.

TURNER, M. G. Landscape Ecology in North America: Past, Present, and Future. Ecology. 86 (8), pp. 1967-1974. 2005.

TURNER, M. G., GARDNER, R. H. \& O'NEILL, R. V. Landscape ecology in theory and practice : pattern and process. Springer-Verlag. New York, Berlin, Heidelberg. 2001.

URBAN, D. L.; O’NEILL, R. V. \& SHUGART Jr., H. H. Landscape ecology. A hierarchical perspective can help scientists understand spatial patterns. BioScience. 37 (2), pp. 119-127. 1987.

YUSTOS, P. S. La conciencia ecológica. El espejo de una civilización suicida. Gazeta de Antropología. 25. 2009. Disponible en: $<$ http://www.ugr.es/ pwlac/G25_39Policarpo_Sanchez_Yustos.html $>$ [Consultado el 16 de mayo del 2011].

WHITTAKER, R. H. Evolution and measurement of species diversity. Taxon. 21 (2/3), pp. 213-251. 1972.

WIENS, J. A. Spatial Scaling in Ecology. Functional Ecology. 3 (4), pp. 385-397. 1989.

WITMAN J. D. \& KAUSTUV, R. Marine macroecology. The University of Chicago Press. London, Chicago. 2009.

WU, J. \& HOBBS, R. Key issues and research priorities in landscape ecology: An idiosyncratic synthesis. Landscape Ecology. 17, pp. 355-365. 2002. 